\title{
An Electrical Stimulation Method to Control Deep Muscle Contraction using Surface Electrodes
}

Hiroki Ohara ( $\square$ ohara.h.af@m.titech.ac.jp )

Tokyo Institute of Technology

Shoichi Hasegawa

Tokyo Institute of Technology

\section{Research Article}

Keywords: functional electrical stimulation (FES), electrical muscle stimulation (EMS).

Posted Date: January 5th, 2022

DOI: https://doi.org/10.21203/rs.3.rs-1201798/v1

License: (9) This work is licensed under a Creative Commons Attribution 4.0 International License. Read Full License 


\section{Abstract}

Conventional EMS technology cannot stimulate deep muscles to induce muscle contraction using surface electrodes. Several treatments use electrical stimulation for various neurological conditions, including stroke and spinal cord injury. One such treatment is functional electrical stimulation (FES), a form of rehabilitation in which electrical muscle stimulation (EMS) is provided while the muscles are being moved. Here, we show whether two interfering electrical stimulation pulses could stimulate the deep muscles of the forearm to control muscle contraction. The results showed that the strongest torques were generated across the subjects when the reference frequency was mid-frequency $(4,000 \mathrm{~Hz})$ and the beat frequencies were low $(20 \mathrm{~Hz}, 40 \mathrm{~Hz}, 80 \mathrm{~Hz}, 160 \mathrm{~Hz}$ and $320 \mathrm{~Hz})$. This study is the first counterexample to demonstrate that it is possible to control muscle contraction in the deep muscles of the forearm using surface electrodes, which was previously thought to be impossible.

\section{Introduction}

Electrical muscle stimulation (EMS) has a long history; however, the method for the contraction of deep muscles using surface electrodes has not yet been established yet ${ }^{1}$. Research into the use of EMS for therapy and rehabilitation has been ongoing for greater than 100 years ${ }^{2}$. In recent years, several studies have shown that EMS is an effective tool for the treatment of patients with spinal cord injuries and neurological disorders ${ }^{3,4}$. Functional electrical stimulation (FES), i.e. the application of EMS and physical movement simultaneously, has been highlighted as a promising method of rehabilitation ${ }^{5-7}$. Additionally, in the field of human-computer interaction, EMS has attracted attention as a haptic interface ${ }^{8}$. Thus, the safety and reproducibility of EMS technology must be refined. If EMS were to be used daily, surface electrodes would be superior to implantable electrodes in terms of less burden on the user. However, surface electrodes are inferior in their ability to stimulate nerves and muscles selectively ${ }^{9}$. Moreover, when electrical stimulation is applied percutaneously, the control of contractions in deep muscles is challenging.

Several studies have highlighted methods for the application of electrical stimulation deep in the human body through varying the parameters of the stimulus pulse. Furthermore, previous studies revealed that four parameters of electrical stimulation are essential to change the stimulation point ${ }^{10}$. These are 'waveform', 'frequency', 'amplitude' and 'pulse width'1,11 (except in special cases, such as 'Russian Current ${ }^{\prime 12}$, etc.). Of these factors, the waveform and frequency are essential in determining the depth of the stimulation. Previous studies have suggested that square waves do not penetrate deep muscles well, whereas sine waves pass through adipose tissue and reach deep muscles more efficiently ${ }^{13,14}$. Regarding the relationship between frequency and skin resistance, Rosell et al. highlighted that the higher the frequency, the lower the resistance of the $\operatorname{skin}^{15}$. However, conventional EMS is achieved using lowfrequency stimulus pulses, and high-frequency pulses do not provide adequate muscle contraction. Furthermore, in addition to the parameters, the placement of the electrodes has a significant influence on the stimulation ${ }^{13,16}$. 
Interferential current stimulation (ICS) is a method used for the electrical stimulation of deep muscles. ICS originated from 'interferential therapy', which was used by Dr Hans in the 1950s for cosmetic, rehabilitation and pain relief purposes ${ }^{17}$. As the name suggests, two independent sources of electrical stimulation generate interference waves in the tissue. ICS has been shown to stimulate tissues more deeply than other stimulation methods ${ }^{17,18}$, which is plausible as both currents are sinusoidal and of midfrequency. Several studies have demonstrated that ICS can be used to relieve muscle pain and induce slight muscle contractions in the quadriceps muscles ${ }^{19-21}$. The quadriceps muscles that can be stimulated with ICS are located in the superficial layers beneath the skin and fat ${ }^{22,23}$. Although ICS can stimulate deep tissue, the conditions necessary for ICS to induce deep muscle contraction are not yet clear.

We hypothesised that maximum contraction of deep muscle could be triggered when the beat frequency of ICS was close to the low frequency used in EMS. We termed this method 'Electrical deep Muscle Stimulation (EdMS)'. To test this hypothesis, we applied electrical stimulation to the pronator teres muscle (a deep muscle ${ }^{22,23}$ ) in the forearm by varying the beat frequency from $20 \mathrm{~Hz}$ to $2,560 \mathrm{~Hz}$. The forearm torque associated with the contraction of the pronator teres muscle was measured with a force sensor to assess whether the forearm was pronated or not. The position of the electrodes and the maximum voltage were calibrated to each participant, and the other parameters of the stimulus pulse (sinusoidal and biphasic) were not altered.

As hypothesised, the maximum forearm torque occurred when the beat frequency was $80 \mathrm{~Hz}$. In addition to the main findings obtained via EMS, we also attempted to show that the proposed method (EdMS) is superior to other methods. When the stimulus pulse (which is rectangular, biphasic and low frequency) used in conventional EMS was applied using the same electrode configuration as in EdMS, no rotational movement of the forearm was induced. Furthermore, using three different frequencies of electrical stimulation $(100 \mathrm{~Hz}, 4,000 \mathrm{~Hz}$ and $10,000 \mathrm{~Hz})$ with a non-interfering sinusoidal wave, significantly less forearm torque was induced compared to that induced via EdMS. These results suggest that, in general, the electrical stimulation that causes muscle contraction is lower in frequency and is independent of the muscle layer.

\section{Results}

\%MVIC. We measured the maximum voluntary isometric contraction (MVIC) of the pronator teres muscle of the participants and obtained a percentage of force/torque generated by electrical stimulation to the MVIC (\%MVIC). The MVIC of the pronator teres muscle was referenced to the force sensor values and, overall, the torque showed a mean of $1.30 \mathrm{~N} / \mathrm{m}$ and a standard deviation of $0.576 \mathrm{~N} / \mathrm{m}(n=13)$.

Sinusoidal stimulus pulses with a beat. To investigate the effect of the beat frequency on muscle contraction, each participant was simultaneously exposed to two types of electrical stimulation: stimulation pulses at reference frequencies $(100 \mathrm{~Hz}, 4,000 \mathrm{~Hz}$ and $10,000 \mathrm{~Hz})$ and stimulation pulses discretely deviated from the reference frequency by $20 \mathrm{~Hz}$ to $2,560 \mathrm{~Hz}$. Initially, the reference frequency 
was set at $4,000 \mathrm{~Hz}$ (mid-frequency), which is more likely to stimulate deep muscles, and the torque was measured when the beat frequency was discretely varied from $20 \mathrm{~Hz}$ to $2,560 \mathrm{~Hz}$ (one-way repeated measures ANOVA, $F=11.8, p=0.00131)$. Figure 1 illustrates that, overall, the maximum torque was generated when the beat frequency was $80 \mathrm{~Hz}$ (median \%MVIC $=24.7 \%$ ). A two-sided paired t-test conducted at each beat frequency showed that each of the lower frequencies $(20 \mathrm{~Hz}, 40 \mathrm{~Hz}, 80 \mathrm{~Hz}, 160$ $\mathrm{Hz}$ and $320 \mathrm{~Hz}$ ) differed significantly from the higher frequencies $(640 \mathrm{~Hz}, 1,280 \mathrm{~Hz}$ and 2,560 Hz) (Figure 1 and Table 1). In the following sections, the condition with a reference frequency of $4,000 \mathrm{~Hz}$ and beat frequencies of $20 \mathrm{~Hz}$ or $40 \mathrm{~Hz}, 80 \mathrm{~Hz}, 160 \mathrm{~Hz}$ and $320 \mathrm{~Hz}$ is referred to as 'EdMS condition'.

Table 1

Median and two-sided paired t-test for the EdMS conditions at a reference frequency of $4000 \mathrm{~Hz}$.

\begin{tabular}{|llllll|}
\hline Beat frequency [Hz] & Median of \%MVIC [\%] & p-value & \\
\hline & & Beat freq. $=$ & $640 \mathrm{~Hz}$ & $1,280 \mathrm{~Hz}$ & $2,560 \mathrm{~Hz}$ \\
\hline 40 & 22.8 & 0.0137 & 0.00380 & 0.00219 \\
\hline 80 & 24.3 & 0.0114 & 0.00317 & 0.00186 \\
\hline 160 & 24.7 & 0.0122 & 0.00314 & 0.00185 \\
\hline 320 & 23.1 & 0.00796 & 0.00179 & 0.000836 \\
\hline
\end{tabular}

We also interfered stimulus pulses when the reference frequency was $100 \mathrm{~Hz}$ (low frequency) and 10,000 $\mathrm{Hz}$ (high frequency) to assess whether torque was generated. An interaction was observed among the different reference frequencies (two-way repeated-measures $\operatorname{ANOVA}, F(2,24)=21.7, p=0.000250$ ) and the different beat frequencies (two-way repeated-measures ANOVA, $F(7,84)=11.3, p=0.00119$ ). When the reference frequency was $100 \mathrm{~Hz}$ (one-way repeated measures ANOVA, $F=1.14, p=0.325$ ), the \%MVIC was highest when the beat frequency was $1,280 \mathrm{~Hz}$ (median \%MVIC $=0.893 \%$ ). A significant difference in $\% \mathrm{MVIC}$ was observed between the conditions (reference frequency: $100 \mathrm{~Hz}$, beat frequency: $1,280 \mathrm{~Hz}$ ) and each of the EdMS conditions. When the reference frequency was $10,000 \mathrm{~Hz}$ (one-way repeated measures ANOVA, $F=0.640, p=0.631$ ), the \%MVIC was highest when the beat frequency was $40 \mathrm{~Hz}$ (median \%MVIC $=0.167 \%$ ). A significant difference in MVIC was observed between the conditions (reference frequency: 10,000 Hz, beat frequency: $40 \mathrm{~Hz}$ ) and each of the EdMS conditions.

As most of the previous studies of EMS using surface electrodes focused on pain, we used a questionnaire to assess whether pain was felt through our method. The mean of the faces rating scale (FRS) at each reference frequency was $3.62(\mathrm{SD}=1.89)$ at $100 \mathrm{~Hz}, 3.49(\mathrm{SD}=1.84)$ at 4,000 Hz and 1.93 $(S D=1.50)$ at $10,000 \mathrm{~Hz}$. A significant difference was observed among the reference frequencies (twoway repeated-measures ANOVA, $F(2,24)=6.10, p=0.0135)$. However, no significant differences were observed among the beat frequencies (two-way repeated-measures ANOVA, $F(7,84)=0.517, p=0.578$ ), and no interaction was observed between the reference frequency and the beat frequency (two-way 
repeated-measures ANOVA, $F(14,168)=1.73, p=0.206)$. Additionally, no significant difference was observed in the beat frequency at the reference frequency of $4,000 \mathrm{~Hz}$, where a large \%MVIC is more likely to occur (one-way repeated measures ANOVA, $F=0.867, p=0.456$ ).

Sinusoidal stimulus pulses without a beat. To investigate whether the beat frequency significantly affected the contraction of the pronator teres muscle, we measured the torque when a single stimulus pulse or the same frequency stimulus pulses were applied. A single electrical stimulation pulse from the top set of electrodes resulted in the largest $\% \mathrm{MVIC}$ of $4,000 \mathrm{~Hz}$ (median $\% \mathrm{MVIC}=0.750 \%$ ) (one-way repeated measures ANOVA, $F=4.71, p$-value $=0.0407$ ). Additionally, a single electrical stimulation pulse from the lower set of electrodes resulted in the largest \%MVIC of $4,000 \mathrm{~Hz}$ (median \%MVIC $=0.0602 \%$ ) (one-way repeated measures ANOVA, $F=0.806, p$-value $=0.443$ ). When two stimulus pulses of the same frequency were applied, the median \%MVIC was negative for all conditions and the absolute value of \% was a maximum at $10,000 \mathrm{~Hz}$. (median $\% \mathrm{MVIC}=-0.292 \%$ ) (one-way repeated measures ANOVA, $\mathrm{F}=$ $0.0719, p=0.931)$. These two conditions resulted in smaller \%MVIC values than each of the EdMS conditions.

EMS conventional stimulus pulse. As the pronator teres muscle is partially located in the superficial muscle, we investigated whether torque could be induced by conventional EMS. The median torque was measured at $0.0486 \mathrm{~N} / \mathrm{m}$ when the stimulation pulse was applied from the upper set of electrodes and $-0.0672 \mathrm{~N} / \mathrm{m}$ when the stimulation pulse was applied from the lower set of electrodes (two-sided paired ttest, $p=0.612$ ). For the conventional parameters, the \%MVIC value was smaller than that of each of the EdMS conditions.

\section{Discussion}

In the present study, our hypothesis was proven and the results obtained were statistically superior to sinusoidal electrical stimulus pulses without a beat and conventional EMS when conditions such as the voltage and placement of electrodes (Figure 2) were the same. As the pronator teres muscle could not be stimulated using conventional EMS stimulus pulses, we can conclude that EdMS stimulated it at a deeper layer. Additionally, a beat frequency of $0 \mathrm{~Hz}$ corresponded to a doubling of the amplitude at the point of interference. Little torque was generated when the beat frequency was $0 \mathrm{~Hz}$, which suggests that the interference may have caused muscle contraction in the deeper muscles. More significant differences were observed in the torque values at low beat frequencies $(20 \mathrm{~Hz}, 40 \mathrm{~Hz}, 80 \mathrm{~Hz}$ and $160 \mathrm{~Hz})$ compared with the medium beat frequencies $(640 \mathrm{~Hz}, 1,280 \mathrm{~Hz}$ and 2,560 Hz). Considering that low frequencies are often used in conventional EMS, it can be assumed that the parameters of the stimulation pulse that causes muscle contraction are the same for the superficial muscles and deep muscles.

We demonstrated that EdMS is only effective for the pronator teres muscle; however, this method may also be applicable for the supinator muscle and the deep muscles of the fingers. The supinator muscle is located at a deeper level than the pronator teres muscle and has the function of supinating the forearm ${ }^{22,23}$. Since the higher the frequency of the stimulation pulse, the higher the possibility of 
stimulating the deeper layer ${ }^{15}$, we supposed that a frequency greater than $4,000 \mathrm{~Hz}$ (which we used in our experiment) could control the contraction of the supinator muscle. We demonstrated that we could control the contraction of the pronator teres muscle, thus, if we could control the contraction of the supinator muscle, we would be able to control all wrist movements via the surface electrodes. The muscles that move the DIP, PIP and MP joints of the thumb and index finger are located in the deep layers ${ }^{22,23}$. However, as the finger muscles are densely packed at the wrist, the interference points must be extremely precise to be controlled by EdMS. It is also believed that when EdMS is applied to muscles other than the pronator teres muscle, the size and placement of the electrodes will change.

The size of the electrodes and the distance between them will determine where the stimulation pulse reaches and how painful the stimulation pulse will be. When stimulation pulses are applied from surface electrodes, it is difficult to measure the voltage distribution under the skin ${ }^{24}$. Therefore, the location of the point of interference between multiple stimulation pulses is ambiguous. Several studies have been conducted in an attempt to control this interference point via the application of stimulation pulses from multi-channel surface electrodes ${ }^{25,26}$. However, this method is not suitable for deep muscles because the electrode size is too small ${ }^{27,28}$. Using EdMS, however, we were able to contribute to the reduction of the electrode size by increasing the frequency of the stimulation pulses. This facilitated the control of the superficial muscles and the deep muscles while the electrodes were organised in an array. Additionally, the smaller the electrode size in conventional EMS, the greater the pain felt, due to the resistance of the $\operatorname{skin}^{16,29,30}$. This can be resolved by increasing the frequency of the stimulation pulses; however, this method of stimulating the superficial muscles at a high frequency remains unclear and will be an issue in the future.

Despite the promising results obtained during this study, two challenges remain, i.e. the calibration of the electrode placement and pain relief. The time required to calibrate the placement of the electrodes varies greatly from person to person, as deep muscles are difficult to palpate and the position of the surface electrodes relative to the muscle changes with body movement as the muscle contracts through electrical stimulation. As a measure of the success of the calibration, we focused on the \%MVIC and FRS. For the parameter with the highest median torque (reference frequency: $4,000 \mathrm{~Hz}$, beat frequency: $80 \mathrm{~Hz}$ ), there were three participants whose \%MVIC was below $5 \%$. Two participants showed an FRS at the reference frequency of $4,000 \mathrm{~Hz}$ that was greater than the FRS at the other reference frequencies and greater than the mean of the overall FRS at the reference frequency of $4,000 \mathrm{~Hz}$. This suggests that other deep nerves and muscles may have been stimulated. The remaining participant had a lower FRS at all reference frequencies compared with the overall average, suggesting that the voltage of the stimulus pulse was not sufficient. To solve the calibration issue, we suppose that the search time can be reduced through the use of an electrode array.

The results of the questionnaire did not reveal any significant difference regarding EdMS pain; however, we believe this could be improved via calibration of the placement of the electrodes and modification of the experimental procedure. In this study, the reference frequency was varied between $100 \mathrm{~Hz}, 4,000 \mathrm{~Hz}$ 
and $10,000 \mathrm{~Hz}$, and the questionnaire was compiled while the beat frequency was varied from $0 \mathrm{~Hz}$ to $2,560 \mathrm{~Hz}$ at a single reference frequency. The higher the frequency, the less painful the stimulus ${ }^{15,30}$. Moreover, if the subject becomes accustomed to the electrical stimulation during the experiment, it becomes difficult to measure the FRS consistently. Therefore, to measure the FRS, it is necessary to present stimulus pulses of randomly selected parameters at sufficient intervals. We supposed that the FRS would be smaller when the reference frequency was at a mid-frequency rather than a low frequency. However, the FRS did not decrease, partly because many of the subjects were not used to EMS.

Additionally, since many of the subjects declared that they had difficulty judging the sensation of pain, we believe that the results regarding pain could be changed by devising a different experimental method.

\section{Methods}

Participants. Thirteen healthy individuals (11 males and 2 females, median age: 24, Nationality: Japanese, Nepalese and Chinese) who were unaccustomed to electrical stimulation participated in the experiment (Table 2). Eleven participants were recruited from Tokyo Institute of Technology and two postgraduate students were recruited from other universities. The Human Subject Research Ethics Review Committee at Tokyo Institute of Technology approved the study protocol, and the approval number is 2020255. All research was performed in accordance with relevant guidelines/regulations, and written informed consent was obtained from each participant prior to the initiation of the experiment. No adverse events were associated with this study. Furthermore, the study was a basic experiment conducted on human subjects and was not a clinical trial. 
Table 2

Characteristics of the participants.

\begin{tabular}{|llllll|}
\hline Participant & Gender & Age & $\begin{array}{l}\text { Width of Palm } \\
{[\mathrm{cm}]}\end{array}$ & $\begin{array}{l}\text { Forearm } \\
\text { Circumference [cm] }\end{array}$ & $\begin{array}{l}\text { Voltage of Stimulus } \\
\text { Pulse [V] }\end{array}$ \\
\hline 00 & Male & 23 & 7.5 & 26 & 33.75 \\
\hline 01 & Male & 30 & 8.5 & 25 & 33.75 \\
\hline 02 & Male & 70 & 8.0 & 26 & 45 \\
\hline 03 & Female & 23 & 7.0 & 25 & 48.75 \\
\hline 04 & Male & 24 & 7.0 & 23 & 30 \\
\hline 05 & Male & 30 & 8.0 & 26 & 37.5 \\
\hline 06 & Female & 43 & 7.5 & 23.5 & 45 \\
\hline 07 & Male & 31 & 8.0 & 26.5 & 30 \\
\hline 08 & Male & 23 & 7.5 & 21 & 22.5 \\
\hline 09 & Male & 24 & 8.5 & 25 & 37.5 \\
\hline 10 & Male & 23 & 8.5 & 26.5 & 41.25 \\
\hline 11 & Male & 24 & 8.5 & 28.5 & 37.5 \\
\hline 12 & Male & 37 & 8.0 & 23.5 & 37.5 \\
\hline
\end{tabular}

Electrical stimulation apparatus. We designed an original electric circuit referring 'wavEMS'${ }^{31}$ and newly made circuit boards for the electrical stimulators that were used in the experiments. No commercially available electrical stimulators exist that can be programmed to change the shape of the stimulus pulse to work with external sensors and vary the interference conditions of the stimulation pulses. The performance of the apparatus used in this study is based on a rated voltage of $\pm 60 \mathrm{~V}$ and a rated current of $60 \mathrm{~mA}$. Additionally, referring to IEC 60601-2-10:2012, in the event of any issues exceeding the rated current during the experiment, the fuse would physically be blown to ensure the safety of the participants.

For the experiment, two sets of parameters for the stimulus pulse were prepared. One was a sinusoidal biphasic pulse with a duty ratio of 2:1 and the other was a rectangular biphasic electrical stimulation pulse with a duty ratio of $8: 1$. In this study, we treated the latter as a set of parameters that are conventionally used in EMS.

Regarding the electrodes, we used four gel electrodes (Axelgaard Manufacturing Co., Ltd.), which are considered to be the least painful among the surface electrodes ${ }^{29}$. The size of each electrode was $50 \mathrm{~mm}$ $\times 10 \mathrm{~mm}$. 
Experimental apparatus. Because the only muscles in the forearm that rotate it inwards and outwards are the pronator teres muscle and the supinator muscle, the proposed method was evaluated by measuring the inward and outward forearm torque using a six-axis force sensor (055YA251, Leptrino Inc.) (Figure 3). When the elbow is fixed so that the palm is perpendicular to the ground, the forearm can be rotated by up to $90^{\circ}$ inward and outward, respectively. Therefore, we used a six-axis force sensor to measure how the deep muscle generated the torque through electrical stimulation from a reference position where the palm was perpendicular to the ground. As part of the experiment, we designed a hand holder and attached it to the six-axis sensor. The hand holder was designed so that movements other than that of the palm (such as the elbow and fingertips) were not measured as noise. Additionally, the palm and hand holder were fixed with Velcro so that each participant could rapidly remove them in case of an emergency. An arm cover (SK-491, Komine Co., Ltd.) was used to maintain a horizontal position of the subject's forearm, and it had two additional functions: it allowed for the fixing angle of the elbow and the distance from the hand holder to the elbow to be varied, depending on the participant. The force sensor was attached directly to the steel plate with screws. The arm cover was attached to the aluminium frame and the height was adjusted, then the aluminium frame was attached to the steel plate with screws and the steel plate was fixed to the desk in a vice.

Procedures. The experiment was conducted in four stages: calibration, investigation of the response to conventional EMS, investigation of the response to EMS with/without interference and measurement of the MVIC. The calibration determined the placement of the electrodes and the strength of the voltage. Regardless of which hand is dominant, the right forearm is farther from the heart than the left forearm, thus, the right forearm of the participants was electrically stimulated during the experiment. The arrangement of the four electrodes (two pairs) was chosen so that the proposed method (EdMS) would be the most effective. We were careful to note that as the joints moved, the relative positions of the muscles and skin changed ${ }^{32}$.

Therefore, based on preliminary experiments, the most reproducible arrangement was the one adopted for the proposed placement of the electrodes (Figure 2). Noting that the angle of the elbow affected the ease of stimulation, we also adjusted the angle of the device's arm cover so that the subject's response would be the strongest. The voltage was adopted as the maximum value at which the subject did not feel any discomfort and was fixed at this value throughout the experiment unless any issues arose.

After the calibration was completed, the participants placed their right forearm on the experimental device. They received stimulation pulses of conventional EMS parameters from a pair of electrodes for 1 second and the forearm torque was measured for 2 seconds simultaneously as the stimulation was initiated. Three measurements were taken under each condition, and because there were two pairs of electrodes, a total of six measurements were taken for each subject (Figure 4). The timing of the stimulation was informed by voice so that the subject would not be startled and make unnecessary movements. 
Once the investigation of the response to conventional EMS was completed, the interference-free $(0 \mathrm{~Hz}$ beat frequency) electrical stimulation was analysed. There are two types of interference-free electrical stimulation: (i) a single sinusoidal stimulation pulse, and (ii) two sinusoidal stimulation pulses of the same frequency. In the case of (i), electrical stimuli of $100 \mathrm{~Hz}, 4,000 \mathrm{~Hz}$ and $10,000 \mathrm{~Hz}$ were applied independently from each pair of electrodes three times (18 times in total), and the torque was measured (Figure 4). In the case of (ii), the same three frequencies as in (i) were applied simultaneously from two pairs of electrodes three times ( 9 times in total), and the torque was measured.

After (i) and (ii) were completed, the interference conditions were compared. In the interference condition, the upper electrode pair applied stimulation pulses at the reference frequency, and the lower electrode pair applied stimulation pulses at a frequency that deviated from the reference frequency by a beat frequency. We used the same reference frequencies as in (i) and (ii) $(100 \mathrm{~Hz}, 4,000 \mathrm{~Hz}$ and 10,000 Hz) and the beat frequencies used were $20 \mathrm{~Hz}, 40 \mathrm{~Hz}, 80 \mathrm{~Hz}, 160 \mathrm{~Hz}, 320 \mathrm{~Hz}, 640 \mathrm{~Hz}, 1,280 \mathrm{~Hz}$ and 2,560 Hz. Under the conditions of one reference frequency and one beat frequency, electrical stimulation was applied three times and the torque was measured (72 times in total) (Figures 5, 6 and 7).

As a comparison of the interference conditions had not been conducted previously, the participants were asked to complete a questionnaire for each condition to obtain additional information. Four questions were asked based on the intensity of the pain, the type of pain, sensations other than pain and the sense of agency during electrical stimulation. The participants were asked to respond to the FRS regarding the intensity of the pain, part of the McGill pain questionnaire (MPQ) regarding the type of pain, an openended description regarding the sensations other than pain and the numeric rating scale (NRS) regarding the sense of agency.

Finally, the spontaneous pronation torque of the forearm was measured. No electrical stimulation was applied to the participants. Each participant placed his or her right arm on the experimental apparatus and waited for the sound to be generated at random intervals. When the sound was generated, the participant grasped the handle and pronated it with maximum force by pushing the forearm inwards until the sound faded. The participants were reminded not to use their biceps. The measurements were taken five times per participant, and the maximum torque value obtained was considered to be the MVIC.

Data processing. The data from the six-axis force sensors were initially processed using our original software. When measuring the torque via electrical stimulation, the force sensor values that were measured simultaneously while the stimulation pulse was applied were considered as the baseline and subtracted from the measured forces and torques. Similarly, when measuring the MVIC, the force sensor value that was measured simultaneously as a sound was heard was taken as zero-based. During the experiment, the data were obtained for the forces in the three axes and the torques around the three axes. After the experiment, the values of the rotational torques of the forearm were calculated using a statistical analysis programme that we created. The point of action where the participant's force was applied to the handle was set at the centre of the palm, and the force sensor measured the torque and translational force. The value of the rotational torque of the forearm was calculated for each participant 
by subtracting the torque due to the translational force from the torque around the axis parallel to the forearm. The distance of the torque due to the translational force was calculated as the distance between the centre of the participant's palm and the centre of the force sensor. Due to a large variation in the torque data between the participants, the index (\%MVIC) that normalised the mean value of the torque value via the electric stimulation by MVIC was calculated (Figure 8 ). The average value of the torque due to electrical stimulation was calculated from 0.55 seconds to 1.05 seconds after the electrical stimulation was applied.

Statistical analyses. A two-way repeated-measures ANOVA was performed on the time-series data of the torque measured during electrical stimulation to investigate whether a particular combination of reference and beat frequencies was efficient at stimulating the pronator teres muscle. For each of the other conditions of electrical stimulation, a one-way repeated-measures ANOVA was used to assess whether there was a dominant combination. A two-sided paired t-test was used to show the superiority of each of the EdMS conditions compared with the other parameters. Regarding the FRS of the questionnaire, a non-parametric test (Wilcoxon rank-sum test) was used. We considered $p<0.05$ to be statistically superior.

\section{Declarations}

Code availability. Theanalyses of thedata were conducted using Python, and this information is available from the corresponding author upon request.

Data availability. The data that support the findings of this study are available from the corresponding author upon request.

\section{References}

1. Doucet, B. M., Lam, A. \& Griffin, L. Neuromuscular electrical stimulation for skeletal muscle function.Yale J Biol Med 85(2), 201-215 (2012).

2. Cambridge, N. A. Electrical apparatus used in medicine before 1900. Proc R Soc Med 70(9), 635-641 (1977).

3. Porcari, J. P. et al. The effects of neuromuscular electrical stimulation training on abdominal strength, endurance, and selected anthropometric measures. J Sports Sci Med 4, 66 (2005).

4. Shin, H., Watkins, Z. \& Hu, X. Exploration of hand grasp patterns elicitable through non-invasive proximal nervestimulation. Sci Rep 7, 16595, DOI: https://doi.org/10.1038/s41598-017-16824-1 (2017).

5. Greve, J. et al. Functional electrical stimulation (FES): muscle histochemical analysis. Spinal Cord 31, 764-770, DOI: https://doi.org/10.1038/sc.1993.119 (1993).

6. Biasiucci, A. et al. Brain-actuated functional electrical stimulation elicits lasting arm motor recovery after stroke. Nat Commun 9, 2421, DOI: https://doi.org/10.1038/s41467-018-04673-z (2018). 
7. Selfslagh, A. et al. Non-invasive, brain-controlled functional electrical stimulation for locomotion rehabilitation in individuals with paraplegia. Nat Commun 9, 6782, DOI: https://doi.org/10.1038/s41598-019-43041-9 (2019).

8. Kono, M., Takahashi, T., Nakamura, H., Miyaki, T. \& Rekimoto, J. Design guideline for developing safe systems that apply electricity to the human body. ACM Trans Comput Interact 25, 19:1-19:36, DOI: https://doi.org/10.1145/3184743(2018) (2018).

9. Maleševic, N. M. et al. A multi-pad electrode based functional electrical stimulation system for restoration of grasp. J Neuroeng Rehabilitation 9, 1-12 (2012).

10. Bickel, C., Gregory, C. \& Dean, J. Motor unit recruitment during neuromuscular electrical stimulation: a critical appraisal. Eur J Appl Physiol 111, DOI: https://doi.org/10.1007/s00421-011-2128-4 (2011).

11. Petrofsky, J., Laymon, M., Prowse, M., Gunda, S. \& Batt, J. The transfer of current through skin and muscle during electrical stimulation with sine, square, russian and interferential waveforms. J Med Eng Technol 33, 170-181, DOI: https://doi.org/10.1080/03091900802054580 (2009).

12. Ward, A. \& Shkuratova, N. Russian electrical stimulation: the early experiments. Phys Ther $82,1019-$ 1030, DOI: https://doi.org/10.1093/ptj/82.10.1019 (2002).

13. Petrofsky, J. et al. Estimation of the distribution of intramuscular current during electrical stimulation of the quadriceps muscle. Eur J Appl Physiol 103, 265-273, DOI: https://doi.org/10.1007/s00421008-0700-3 (2008).

14. Petrofsky, J. The effect of the subcutaneous fat on the transfer of current through skin and into muscle. Med Eng Phys 30, 1168-1176, DOI: https://doi.org/10.1016/j.medengphy.2008.02.009 (2008).

15. Rosell, J., Colominas, J., Riu, P., Pallas-Areny, R. \& Webster, J. Skin impedance from 1 hz to $1 \mathrm{mhz}$. IEEE Transactions on Biomed Eng 35, 649-651, DOI: https://doi.org/10.1109/10.4599 (1988).

16. Forrester, B. \& Petrofsky, J. Effect of electrode size, shape, and placement during electrical stimulation. J Appl Res 4 (2004).

17. Ganne, J. Interferential therapy. J Physiother 22, 101-110, DOI: https://doi.org/10.1016/S00049514(14)61005-9 (1975).

18. Goats, G. Interferential current therapy. Br J Sp Med 24, 87-92, DOI: http://dx.doi.org/10.1136/bjsm.24.2.87 (1990).

19. Gundog, M. M., Atamaz, F. M., Kanyilmaz, S. M., Kirazli, Y. M. \& Celepoglu, G. M. Interferential current therapy in patients with knee osteoarthritis. Am J Phys Med Rehabil 91, 107-113, DOI: https://doi.org/10.1097/phm.0b013e3182328687 (2012).

20. Jorge, P. F., Susan Armijo, O., David, J. M. \& Douglas, P. G. Effectiveness of interferential current therapy in the management of musculoskeletal pain: a systematic review and meta-analysis. Phys Ther 90, 1219-1238, DOl: https://doi.org/10.2522/ptj.20090335 (2010).

21. Bennie, S., Petrofsky, J., Nisperos, J., Tsurudome, M. \& Laymon, M. Toward the optimal waveform for electrical stimulation of human muscle. Eur J Appl Physiol 88, 13-19, DOI: https://doi.org/10.1007/s00421-002-0711-4 (2002). 
22. Netter, F. H. Atlas of human anatomy (6th edition) (2014).

23. Rohen, J., Yokochi, C. \& Lütjen-Drecoll, E. Anatomy: a photographic atlas (8th edition) (2016).

24. Adams, G., Harris, R., Woodard, D. \& Dudley, G. Mapping of electrical muscle stimulation using MRI. J Appl Physiol 74, 532-537, DOI: https://doi.org/10.1152/jappl.1993.74.2.532 (1993).

25. Popoví c-Bijelí c, A. et al. Multi-field surface electrode for selective electrical stimulation. Artif Organs 29, 448-452, DOI: https://doi.org/10.1111/j.1525-1594.2005.29075.x (2005).

26. Bounyong, S., Adachi, S., Yoshimoto, T., Ota, T. \& Ozawa, J. Controlling interfered area in interferential current stimulation by electrode-area patterning. In 2016 38th Annual International Conference of the IEEE Engineering in Medicine and Biology Society (EMBC), 1721-1724, DOI:

10.1109/EMBC.2016.7591048 (2016).

27. Kuhn, A., Keller, T., Lawrence, M. \& Morari, M. The influence of electrode size on selectivity and comfort in transcutaneous electrical stimulation of the forearm. IEEE Trans Neural Syst Rehabil Eng 18, 255-262, DOI: https://doi.org/10.1109/tnsre.2009.2039807 (2010).

28. Sha, N. et al. A finite element model to identify electrode influence on current distribution in the skin. Artif Organs 32, 639-643, DOI: https://doi.org/10.1111/j.1525-1594.2008.00615.x (2008).

29. Keller, T. \& Kuhn, A. Electrodes for transcutaneous (surface) electrical stimulation. J Autom Control 18, 35-45, DOI: https://doi.org/10.2298/JAC0802035K (2008).

30. Mason, J. L. \& MacKay, N. A. M. Pain sensations associated with electrocutaneous stimulation. IEEE Transactions on Biomed Eng BME-23, 405-409, DOI: https://doi.org/10.1109/TBME.1976.324652 (1976).

31. Kono, M. \& Rekimoto, J. wavEMS: improving signal variation freedom of electrical muscle stimulation. In 2019 IEEEConference on Virtual Reality and 3D User Interfaces (VR), 1529-1532, DOI: https://doi.org/10.1109/VR.2019.8798102 (2019).

32. Ichikawa, K., Jiang, Y., Sugi, M., Togo, S. \& Yokoi, H. Joint angle based motor point tracking stimulation for surface fes: A study on biceps brachii. Med. Eng. \& Phys. 88, 9-18, DOI: https://doi.org/10.1016/j.medengphy.2020.11.014 (2021).

\section{Figures}




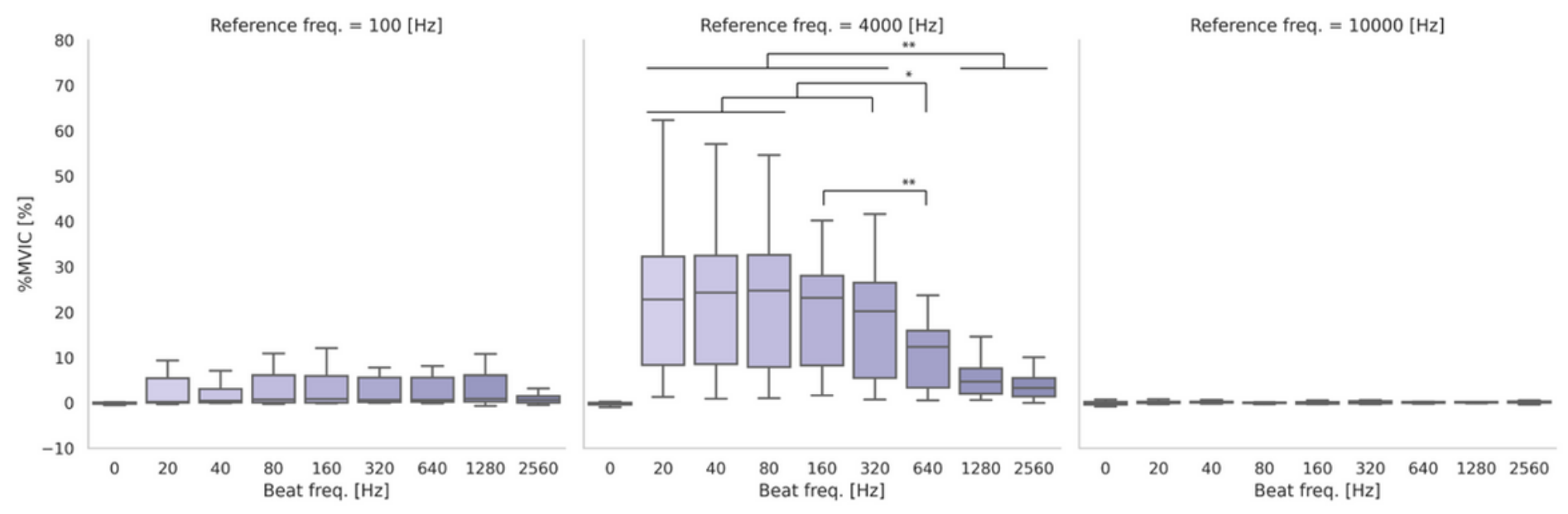

\section{Figure 1}

Relationship between the beat frequency and \%MVIC. The length of the whiskers in a boxplot diagram should be 'less than or equal to 1.5 times the box' on the largest and smallest sides, respectively. The data that exceeds these boundaries are classified as 'outliers'. Significant differences are indicated by an asterisk, where * indicates less than 0.05 and $* \star$ indicates less than 0.001 . 


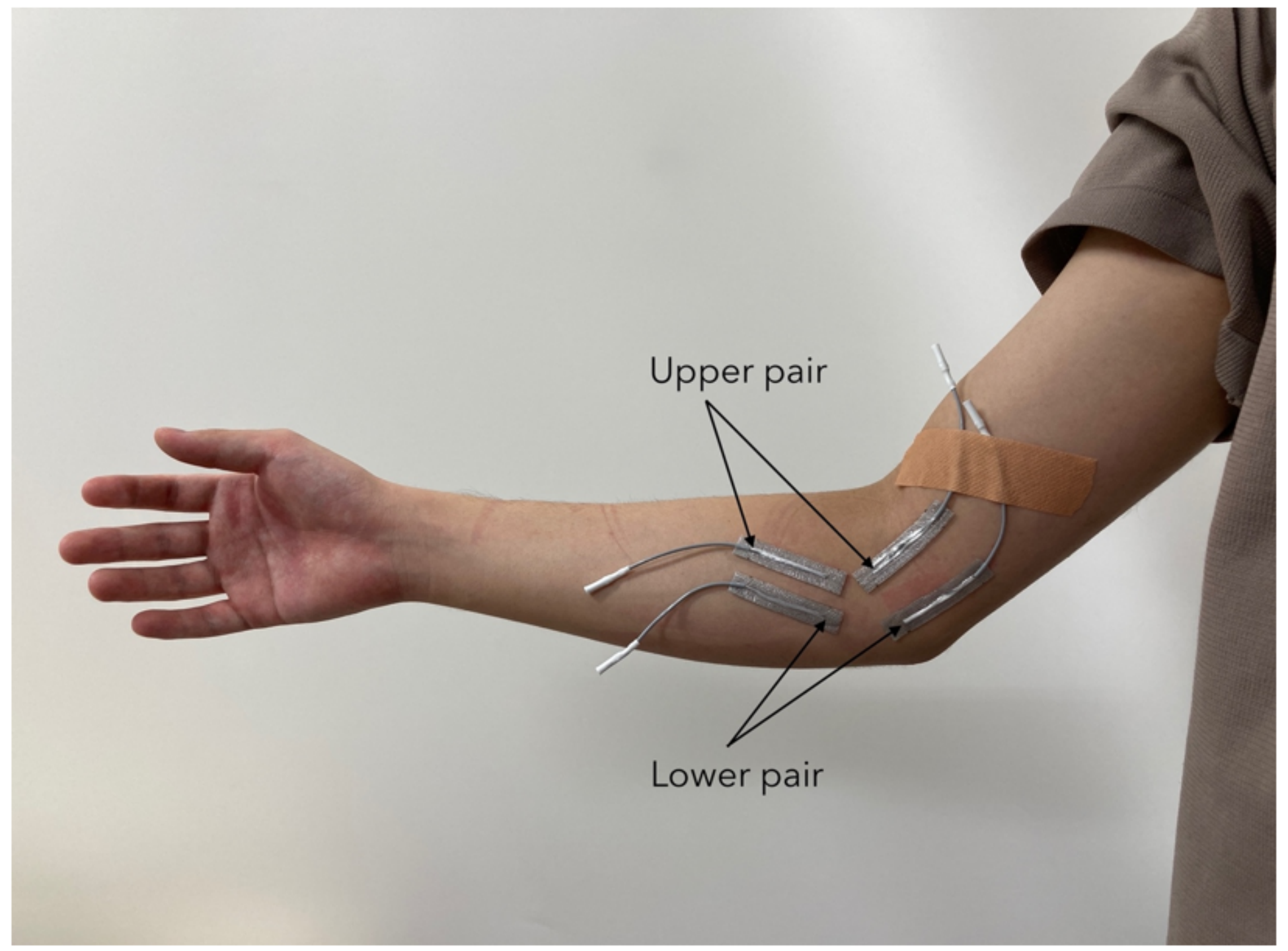

Figure 2

Placement of the electrodes on the arm. 


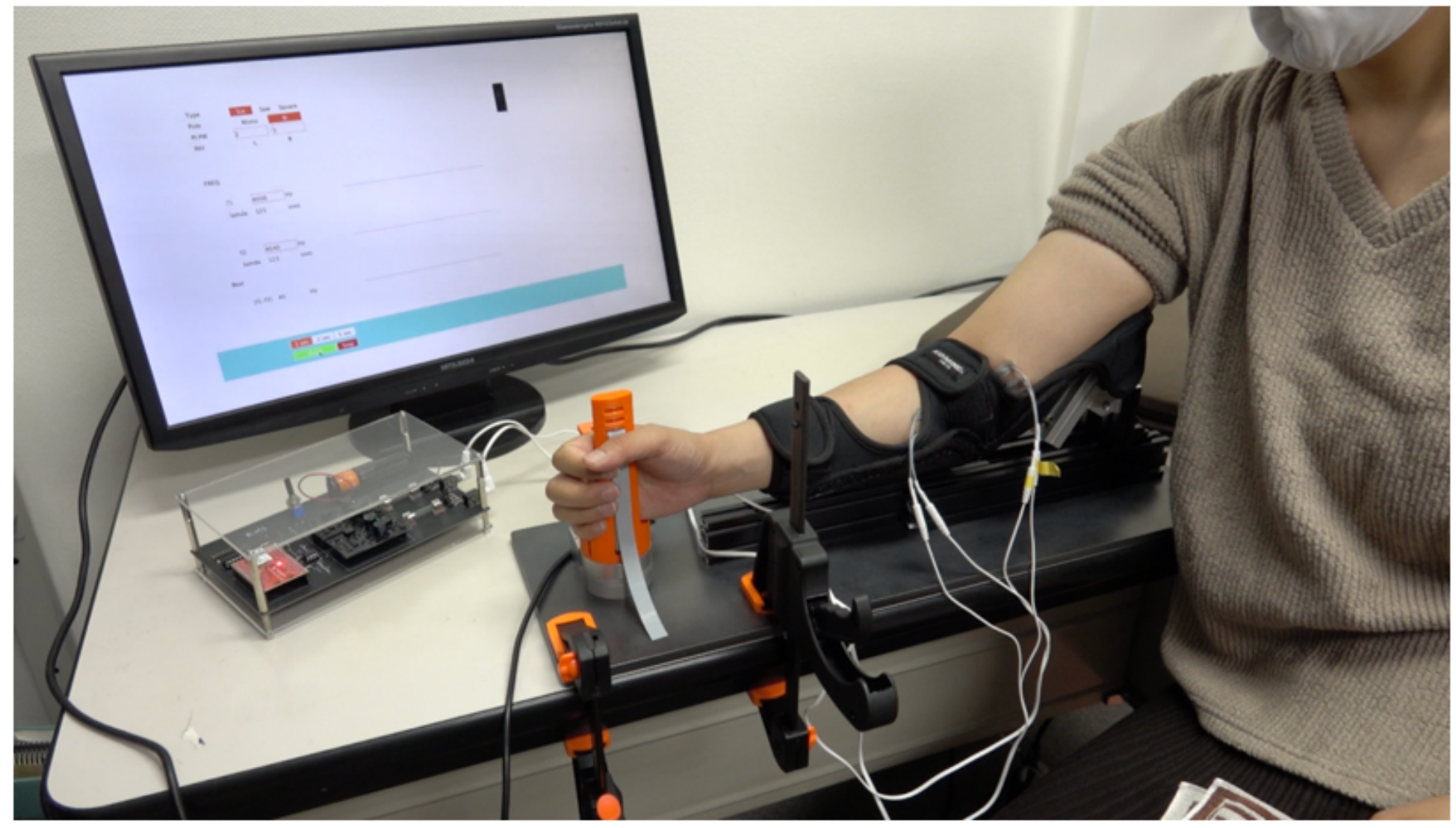

Figure 3

Overview of the experimental setup. 
a
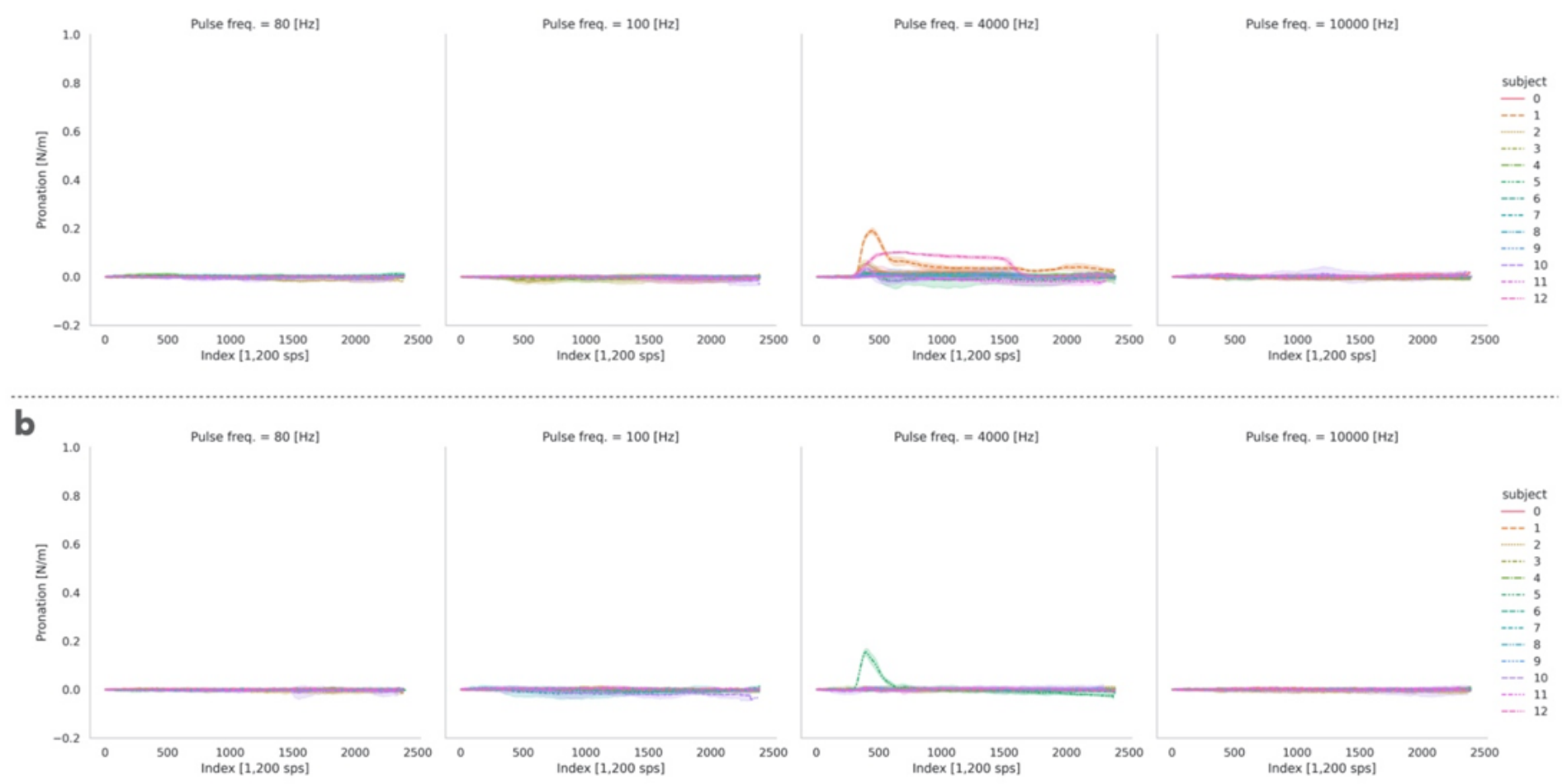

\section{Figure 4}

Temporal changes in pronation of each participant's forearm using conventional EMS and single stimulation pulses. a: The result of the electrical stimulation induced by the 'upper pair' of two pairs of electrodes. The $80 \mathrm{~Hz}$ condition is used in conventional EMS, while $100 \mathrm{~Hz}, 4000 \mathrm{~Hz}$ and $10000 \mathrm{~Hz}$ are sinusoidal stimulation pulses. b: The result of the electrical stimulation induced by the 'lower pair' of two pairs of electrodes. 


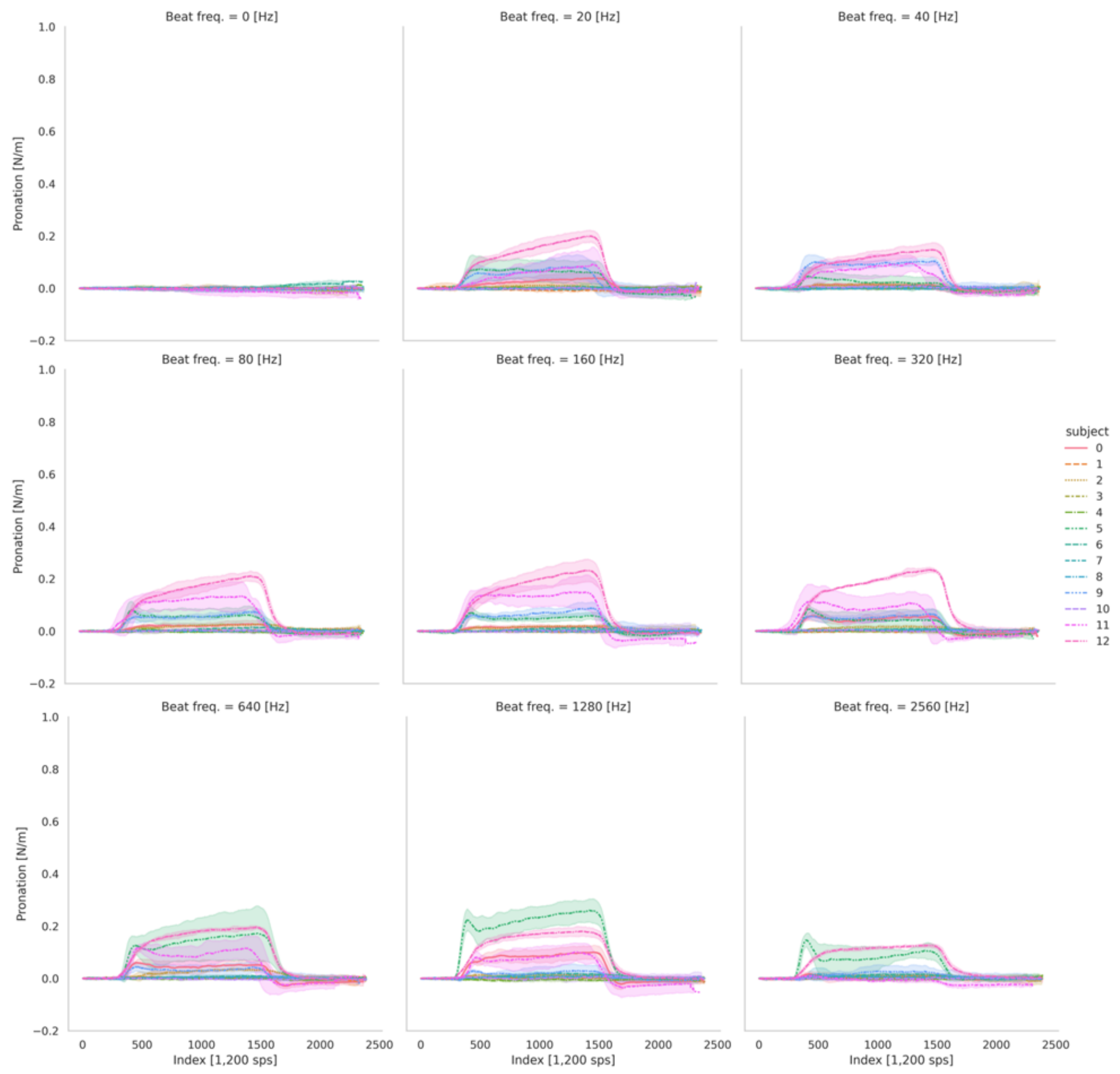

\section{Figure 5}

Temporal changes in the pronation of each participant's forearm at a

reference frequency of $100 \mathrm{~Hz}$ and all beat frequency combinations. The line shows the average of the three measurements, and the light-coloured range shows the standard deviation. 

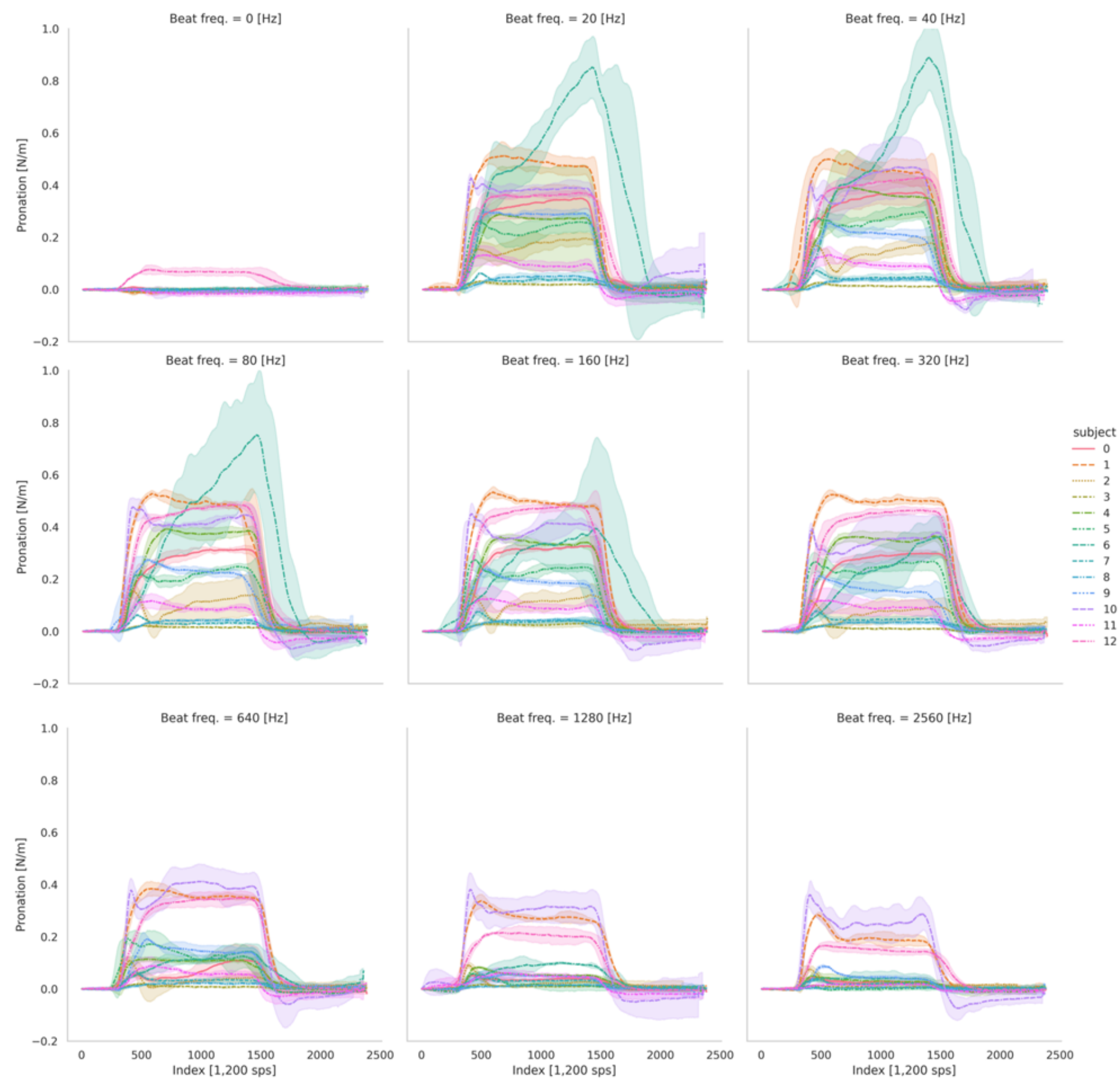

\section{Figure 6}

Temporal changes in the pronation of each participant's forearm at a reference frequency of $4,000 \mathrm{~Hz}$ and all beat frequency combinations. The line shows the average of the three measurements, and the light-coloured range shows the standard deviation. 

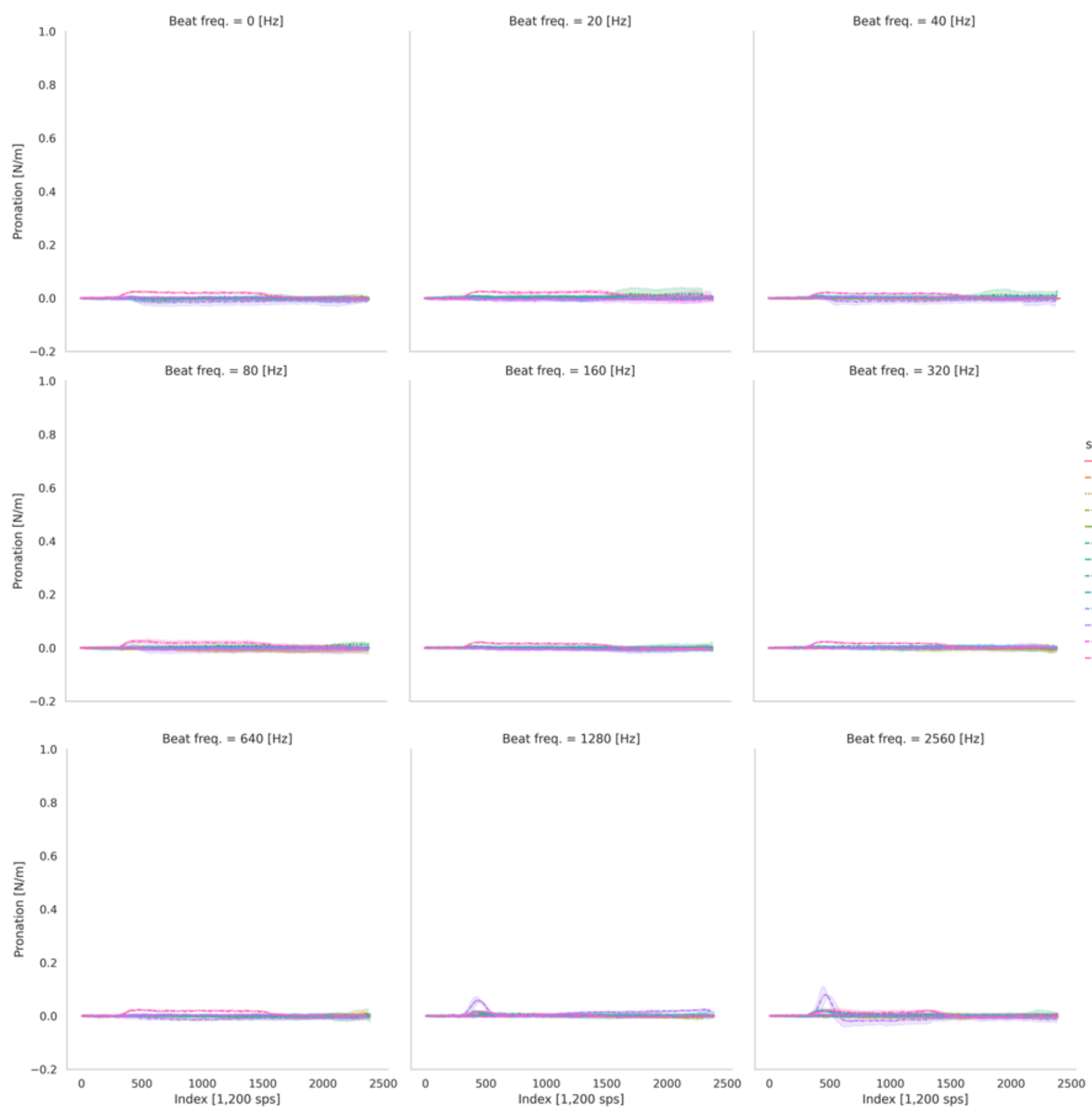

\section{Figure 7}

Temporal changes in the pronation of each participant's forearm at a reference frequency of $10,000 \mathrm{~Hz}$ and all beat frequency combinations. The line shows the average of the three measurements, and the light-coloured range shows the standard deviation. 


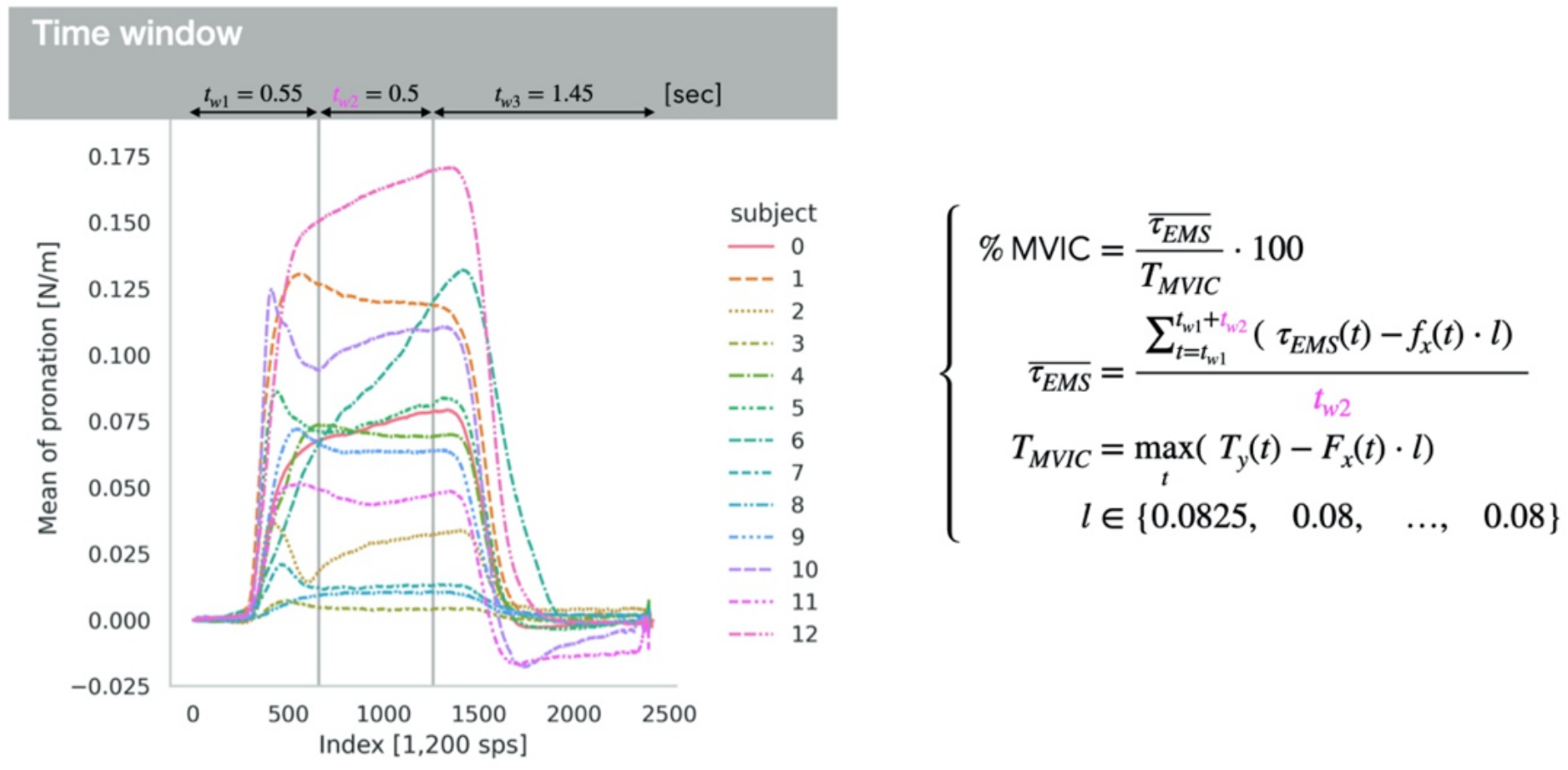

Figure 8

Please see the Manuscript file for the complete figure caption. 